\title{
Erratum to: Least squares moving particle semi-implicit method
}

\author{
Tasuku Tamai · Seiichi Koshizuka
}

Published online: 7 September 2014

(C) OWZ 2014

\section{Erratum to: Comp. Part. Mech. (2014) 1:227-305 DOI 10.1007/s40571-014-0027-2}

The surface detection algorithm described in Sect. 4.3 which includes the adoption of eigenvalues [2] of the renormalization matrix [1] was an original algorithm entirely introduced in [2].

\section{References}

1. Randles P, Libersky L (1996) Smoothed particle hydrodynamics: some recent improvements and applications. Comput Methods Appl Mech Eng 139(1):375-408

2. Marrone S, Colagrossi A, Le Touze D, Graziani G (2010) Fast freesurface detection and level-set function definition in SPH solvers. J Comput Phys 229:3652-3663

The online version of the original article can be found under doi:10.1007/s40571-014-0027-2.

T. Tamai $(\varangle) \cdot$ S. Koshizuka

Graduate School of Engineering, The University of Tokyo,

7-3-1, Hongo, Bunkyo-ku, Tokyo 113-8656, Japan

e-mail: tasuku@mps.q.t.u-tokyo.ac.jp

S. Koshizuka

e-mail:koshizuka@sys.t.u-tokyo.ac.jp 